\title{
M1 inhibition dependency on slowing of muscle relaxation after brief and fast fatiguing repetitive movements: preliminary results
}

\author{
Elena Madinabeitia-Mancebo, Antonio Madrid, Javier Cudeiro, and Pablo Arias
}

NEUROcom (Neuroscience and Motor Control Lab), University of A Coruña, A Coruña, Spain

\begin{abstract}
This work presents preliminary results on the association between central and peripheral expressions of muscle fatigue induced by unresisted repetitive movements. We tested cortico-spinal excitability and intra-cortical inhibition right at the end of $30 \mathrm{~s}$ of maximal rate finger tapping $(f t)$ or after $10 \mathrm{~s}$ of rest; the contractile properties of the muscle were also tested. This procedure was repeated 12 times. In half of the repetitions, the evaluation was done during induced muscle isquemia. In all cases $f t$ rate decreased during the $30 \mathrm{~s}$ of task. Isquemia produced a slowing of muscle contractile properties in all cases, after $f t$ as well as after $10 \mathrm{~s}$ of rest post- $f t$. Intracortical inhibition increased immediately after $f t$, but recovered after $10 \mathrm{~s}$, regardless the presence of muscle isquemia. Our results suggest that the increment of inhibition in M1 after fatiguing repetitive movements is central in origin, and not an adaptation to the slowed contractility of the muscle.
\end{abstract}

\section{Introduction}

Muscle fatigue is common in daily activities and in neurological patients. Muscle fatigue may have central and/or peripheral origin [1]. Central and peripheral expressions of fatigue have been thoroughly studied for isometric activities [1], but for rhythmic repetitive movements (RRM) their peripheral expressions are much more understood that their central mechanisms [2].

Since RRM are essential for daily activities, we have evaluated the possible link between the previously shown increased inhibition of the M1 (central expression of fatigue) [3] and the alteration of muscle contractile properties (peripheral expression of fatigue) [4]. This is a relevant issue since some non-invasive brain stimulation (NIBS) techniques are good candidates to reduce central fatigue during repetitive movements, but their mode of application might vary depending on whether the increased inhibition of M1 after fatiguing RRM is central in origin, or, conversely, reflects a cortical adaptation to a less efficient muscle.

\section{Methods}

\subsection{Participants}

Six young healthy participants took part in the experiments (age range 18-25 yrs, 2 men).

\subsection{Procedure and Analysed Variables}

Participants executed 12 sets of index $f t$ at the maximal possible rate. Ft lasted $30 \mathrm{~s}$, and 3 min and 10 $\mathrm{s}$ was the rest period between sets. 
During each set, $40 \mathrm{~s}$ before $f t$, we tested M1 intracortical inhibition and the slowing of muscle relaxation. For this, subjects executed a maximal voluntary contraction (MVC) with the index finger towards flexion of the metacarpo-phalangal joint in response to a red-LED cue. During MVC, transcranial magnetic stimulation (TMS) was applied on first dorsal interosseous cortical hot-spot, during MVC plateau. The red-LED went off $500 \mathrm{~ms}$ after TMS indicating to the subjects to stop the MVC. After the MVC, with the muscle at rest, we applied percutaneous ulnar nerve stimulation (PNS). It consisted of a $100 \mathrm{~Hz}$-triplet of pulses at supramaximal intensity, which was delivered $3.5 \mathrm{~s}$ after TMS. The acquired variables were the silent period (SP) duration induced by TMS during MVC (reflecting M1-intracortical GABAb inhibition), and the half relaxation time (HRT) of the twitch elicited at the muscle at rest by PNS (reflecting the contractile properties of the muscle; i.e., peripheral fatigue). The recording of these variables at this time before $f t$ is referred as PRE.

Next, after $40 \mathrm{~s}$, subjects performed $f t$ at their maximal possible rate, during $30 \mathrm{~s}$. The median $f t$ rate for the 4 first seconds (PRE) and the 4 last seconds (POST) of the task were considered to evaluate the expressions of fatigue.

Following $f t$, and without resting time, participants executed another MVC in response to a red-LED cue, and stimulation was applied as before $f t$. This was termed POST. In half of the sets, however, a time lag of $10 \mathrm{~s}$ was established between $f t$ and the POST-MVC, during which subjects remained at rest. For this purpose an auditory cue prompted subjects to stop tapping $30 \mathrm{~s}$ after $f t$ started, and the red-LED cue was displayed $10 \mathrm{~s}$ after. So that POST was tested immediately after $f t$ (T0) or $10 \mathrm{~s}$ after (T10). Before each set, participants were told about the kind of set (T0 or T10). Order was counterbalance (Fig. 1).

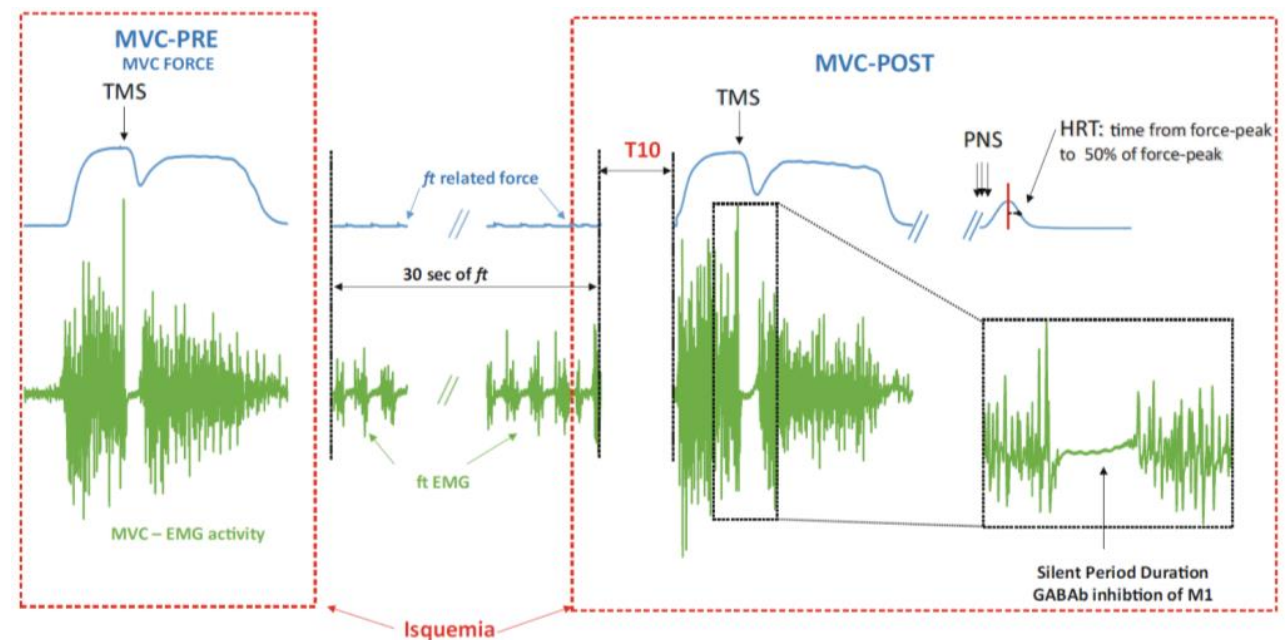

Fig. 1. Representation of the testing procedure in one set. In half of the sets Isquemia was not performed. Also, in half of the set, T10 was replaced with T0.

In half of the sets, and counterbalanced in order, TMS-SP and HRT were tested under isquemia conditions. To do this, a cuff placed around the arm was inflated to $300 \mathrm{~mm} \mathrm{Hg}$ for $20 \mathrm{~s}$, either at PRE and POST. At PRE the cuff was inflated $4 \mathrm{~s}$ before PRE testing; for POST it was inflated $4 \mathrm{~s}$ before the end of $f t$. Therefore, at POST isquemia covered both T0 and T10. The 12 sets were distributed in T0noISQUEMIA, T0-ISQUEMIA, T10-noISQUEMIA, T10-ISQUEMIA, executing 3 of each type counterbalanced in order.

During the whole experiment muscle temperature was monitored (sensor above the skin) and kept constant $\left(\right.$ at $\approx 32{ }^{\circ} \mathrm{C}$ ) by means of a flow of warm/fresh air as needed. 


\subsection{Statistical Analyses}

For all the variables tested, $f t$ rate, TMS-SP duration, and HRT, we used repeated measures ANOVAs with factors TIME (PRE-POST), SET (3 sets), TESTING-LAG (T0-T10) and ISQUEMA LEVEL (with or without isquemia). Significance was set at $\mathrm{p}<0.05$.

\section{Results}

In all sets, we observed a reduction in the $f t$ rate when comparing the first and last $4 \mathrm{~s}$ of the task (PRE and POST, respectively) ANOVA $\mathrm{p}<0.001, f t$ rate drop was about $12 \%$ in magnitude. The effect was evident regardless the presence of isquemia, either for T0 and T10 (Fig. 2). TMS-SP increased $(\approx 10 \%)$ right after $f t$ (POST > PRE) in the T0 sets (ANOVA $\mathrm{p}<0.001$ ), but not in the T10 sets; this was not modified by the presence of isquemia (Fig. 3). The HRT increased under isquemia conditions (ANOVA $p$ $<0.05$ ) and also after $f t$ (i.e., POST, ANOVA p < 0.05), either for T0 and T10 sets (Fig. 4).

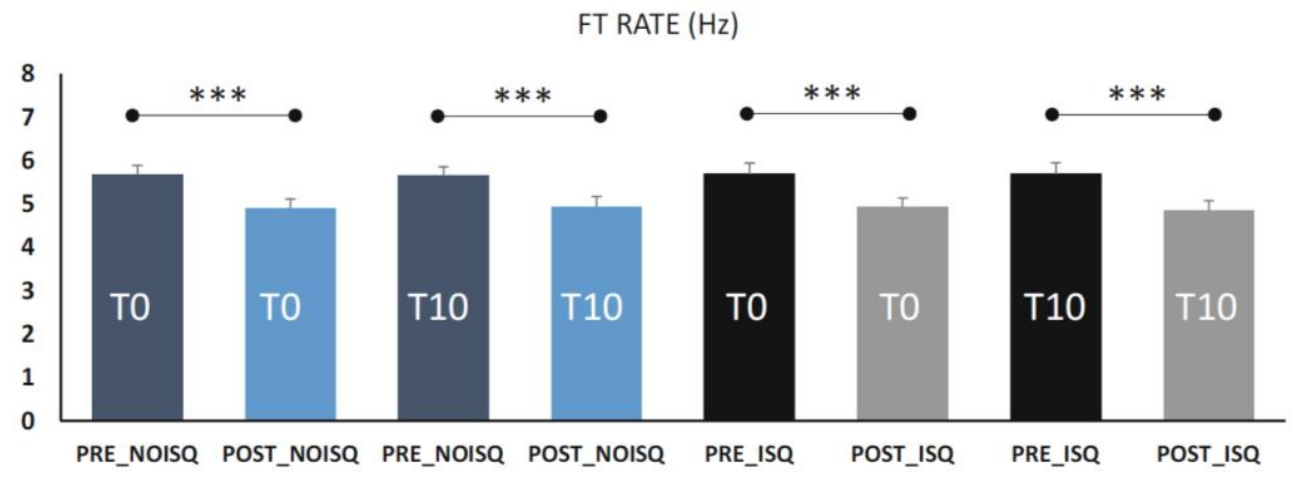

Fig. 2. In all cases, the $f t$ rate decrease from PRE to POST, for clarity sets are presented pooled (set-effect was not significant). $* * * \mathrm{p}<0.001$

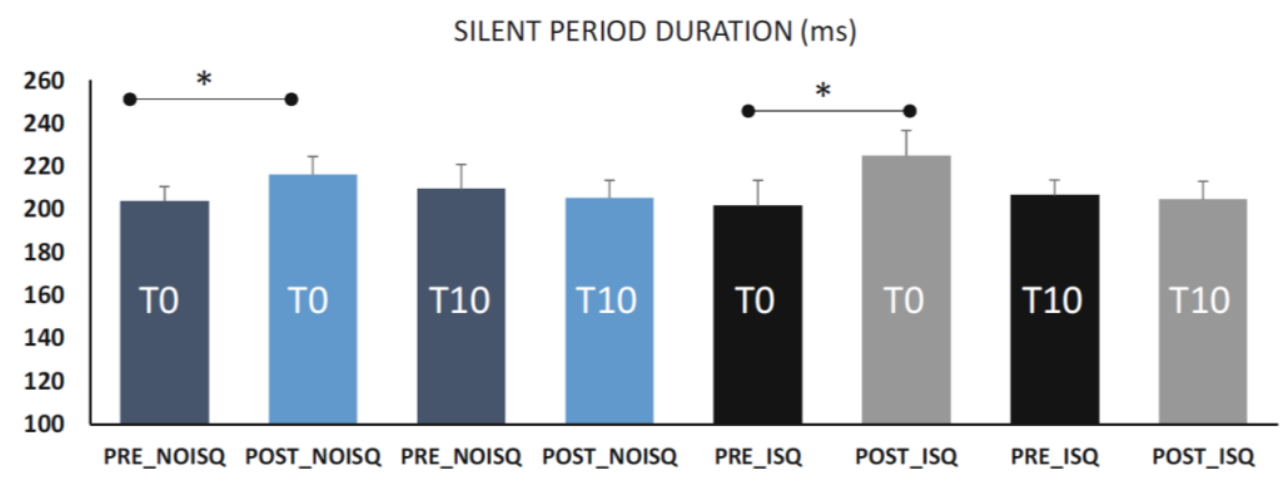

Fig. 3. SP duration increased from PRE to POST-T0 regardless the presence of isquemia. $10 \mathrm{~s}$ of rest after $f t$ induced a recovery of SP, observed at POST-T10. The different sets are presented pooled (set-effect was not significant). ${ }^{*} \mathrm{p}<0.05$. 


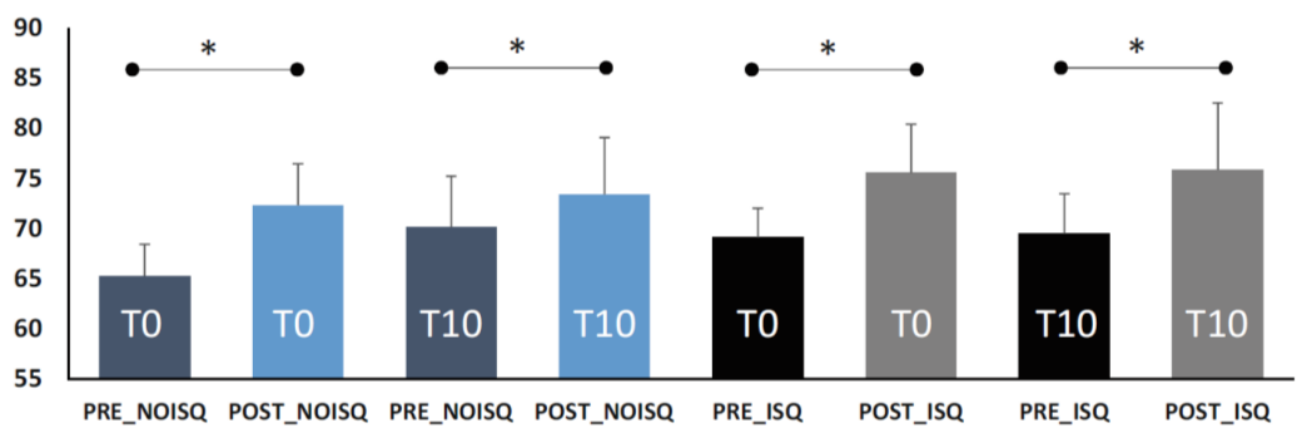

Fig. 4. HRT increased from PRE to POST, regardless T0 or T10. The different sets are presented pooled (seteffect was not significant). *p $<0.05$.

\section{Conclusion}

Our data suggest that the increment of inhibition observed in M1 right after finger tapping at maximal rate, doesn't reflect a central adaptation to the increased slowing of muscle relaxation (i.e. peripheral fatigue) also observed at the end of fatiguing $f$. Therefore, this suggests a central origin of fatigue for this kind of repetitive task. These preliminary results obtained from a small sample $(n=6)$ must be confirmed in studies with larger samples. If they stand, they would advocate the use of NIBS to increase the excitability of the M1 in order to avoid central expressions of fatigue induced by unresisted repetitive movements.

Acknowledgment. This work was supported by Spanish Government (MINECO-2017, DEP2017-87601-R). Pablo Arias is the Project Principal Investigator. Elena Madinabeitia-Mancebo is granted with "Axudas Pre-doutorais" Grant from the Xunta de Galicia, Autonomic Government of Galicia, Spain.

\section{References}

1. Gandevia, S.C.: Spinal and supraspinal factors in human muscle fatigue. Physiol. Rev. 81(4), 17251789 (2001)

2. Allen, D.G., Lamb, G.D., Westerblad, H.: Skeletal muscle fatigue: cellular mechanisms. Physiol. Rev. 88(1), 287-332 (2008)

3. Arias, P., Robles-Garcia, V., Corral-Bergantinos, Y., et al.: Central fatigue induced by shortlasting finger tapping and isometric tasks: a study of silent periods evoked at spinal and supraspinal levels. Neuroscience 305, 316-332 (2015)

4. Jones, D.A.: Changes in the force-velocity relationship of fatigued muscle: implications for power production and possible causes. J. Physiol. 588(Pt 16), 2977-2986 (2010) 